\title{
Fourier-Transformed Local Density of States and Tunneling into a $D$-Wave Superconductor with Bosonic Modes
}

\author{
Jian-Xin Zhu and A. V. Balatsky \\ Theoretical Division, MS B262, Los Alamos National Laboratory, Los Alamos, New Mexico 87545 \\ T. P. Devereaux \\ Department of Physics, University of Waterloo, Ontario, Canada N2L 3GI \\ Qimiao Si \\ Department of Physics \& Astronomy, Rice University, Houston, TX 77005 \\ J. Lee, K. McElroy, and J. C. Davis \\ LASSP, Department of Physics, Cornell University, Ithaca, NY 14850
}

(Dated: May 28, 2005)

\begin{abstract}
We analyze the effects of the electronic coupling to bosonic modes in a d-wave superconductor. The role of the scattering due to boson on the momentum transfer between electronic states in the Brilloine zone is addressed. We consider specific examples of $B_{1 g}$ phonon, breathing mode phonon and spin resonance at $(\pi, \pi)$. The Fourier spectrum of the energy derivative local density of states (LDOS) is calculated. To properly calibrate the effects of different modes we fix the quasipartilce renormalization at specific momentum points. It is found that the $B_{1 g}$ mode with highly anisotropic momentum-dependent coupling matrix element gives rise to well definded features in the Fourier spectrum, at the energy of mode plus gap, with a momentum transfer along the $\mathrm{Cu}-\mathrm{O}$ bond direction of cuprates. This result is in a striking contrast to the cases of the coupling to other modes and also to the case of no mode coupling. The origin of this difference is explored in detail. A comparison with the recent STM experiments is briefly discussed.

PACS numbers: 74.25.Jb, 74.50.+r, 74.20.-z, 73.20.Hb
\end{abstract}

\section{INTRODUCTION}

Determining the nature of single particle excitations is of fundmental importance in our understanding of the superconductivity in high- $T_{c}$ cuprates. To address this issue, a number of spectroscopies have been extensively used, including the angle resolved photoemission spectroscopy (ARPES) and tunneling. The salient features observed in ARPES include: (a) Near the $(\pi, 0)$ $(M)$ point in the Brillouin zone, the spectral function in the superconducting state shows an anomalous line shape, the so-called "peak-dip-hump" structure, (b) Near the $d$-wave node of the superconducting gap, the dispersion shows a characteristic "kink" near 50-70 meV 4.5.6.7.8 Recent ARPES experiments with improved resolution ${ }^{9.10,11.12}$ have revealed another "kink" in dispersion of the antinodal electronic states, near the $M$ point. An unusual spectral dip-hump features similar to the ARPES spectrum have also been observed in the tunneling data $\frac{13.14 .15,16,17,18,19,20}{}$ All these features were suggested to indicate that the electron self-energy renormalization could be due to the electronic coupling to a bosonic mode. Two main scenarios have been presented to explain the experimental data. On one hand, the antinodal renormalization is found to be strongly enhanced below $T_{c}{ }^{9.10 .11}$ Such a strong temperature dependence and the dominance of the coupling strength near the $M$ point can be thought of as evidence for the coupling of electrons to the $41 \mathrm{meV}$ spin resonance mode

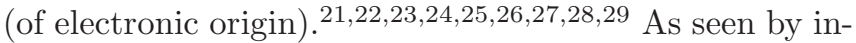
elastic neutron scattering experiments in most of the

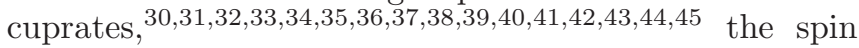
mode intensity substantially turns on below $T_{c}$ (even though some intensity might be present in a normal state) and has a well-defined momentum of $(\pi, \pi)$. This scenario of the electronic coupling to spin resonance mode has also been used to explain the tunneling spectra in planar tunnel junctions. On the other hand, it has been suggested that a significant electronic coupling to the half-breathing in-plane $\mathrm{Cu}-\mathrm{O}$ bond stretching phonon or to the out-of-plane out-of-phase $\mathrm{O}$ buckling $B_{1 g}$ phonon, with an energy of approximately 70 and $35 \mathrm{meV}$, respectively, might be responsible for the dispersion anomalies at the nodal $\underline{\underline{6}}$ and antinodal directions,$\frac{12}{2}$ respectively. These two phonon modes have shown strong lineshape renormalizations with doping and temperature in $\mathrm{Ra}$ man and neutron measurements $\frac{46.47 .48,49,50.51,52.53}{\text { The }}$ advantage of this scenario is that it could naturally explain the band renormalization effect in materials where no spin resonance mode has been detected, in the normal state, and in the deeply overdoped region where the spin mode is neither expected nor observed. To be consistent with ARPES data, this scenario requires the electronphonon interaction to be highly anisotropic ${ }^{54.55}$ and its impact on the electrons to be strongly enhanced in the superconducting state.

The nature of the involved bosonic modes, being phononic or electronic and their role in the mechanism 
of superconductivity remains controversial. Detailed energy and momentum spectoscopy of the relevant bosonic modes might be very helpful in understanding the mechanism of superconductivity in high- $T_{c}$ cuprates. One of the direct spectroscopies that allows energy determination is Inelastic Electron Tunneling Spectroscopy (IETS). It is a well-established and powerful tool that allows the measurement of the characteristic energies of extended modes. Examples of applications of this technique, among many, include measurements of molecular stretching and vibrational modes in metal-insulatormetal tunnel junctions; $\frac{56.57}{5}$ observation of the collective magnetic resonance in tunneling in the superconducting state of high- $T_{c}$ materials ${ }^{20.23}$ and observation of the tunneling features at energies that correspond to the phonon peaks, as seen in planar tunneling into superconductors $\frac{58}{5}$

The IETS directly measures excitation energies. When electrons scatter off a collective mode, a contribution to the electron self energy occurs above a corresponding threshold value of the frequency determined by the mode frequency. Thus, in a tunneling experiment, for bias voltages exceeding the threshold, electrons can excite the mode. This additional scattering channel leads to a step in the density of states (DOS) and in the tunneling conductance. Low temperatures are required to avoid thermal smearing of the step in the conductance. The crucial quantity that reveals inelastic peaks is a second derivative of tunneling conductance with respect to bias voltage: $\frac{d^{2} I}{d V^{2}}(\mathrm{eV})$. Peaks in this quantity are shown to be connected to the energies of the modes, e.g peaks in phonon Density of States $\underline{58}$

In high- $T_{c}$ materials the energies of a number of collective modes, like phonon and spin modes are close. For example the $B_{1 g}$ phonon mode has typical energy of 36$40 \mathrm{meV}$ and spin resonance mode has an energy that ranges between 35-40 meV depending on doping. Hence discrimination between different modes based on only the energy of the observed mode is a challenge. Aside from energy resolved features, it would be useful to come up with the measurement that would allow one to measure typical momenta involved in the electron scattering in cuprates. The ARPES is one such spectroscopy.

Scanning Tunneling Spectroscopy (STM) is another technique that would allow one to resolve the momentum transfer between different electronic states. Recently, the Fourier-transformed scanning tunneling microscopy (FTSTM $)_{59,60}$ was introduced to map out the Fermi surface and the momentum dependence of the $d$-wave energy gap. The results are in good agreement with the ARPES data. While the ARPES provides the information about the energy dispersion of single-particle electronic states, the FT-STM probes the scattering processes between states with different in plane momenta.

In this paper, we show how another technique, the Fourier Transform Inelastic Electron Tunneling Spectroscopy (FT-IETS), would allow simultaneous momentum and energy resolutions of the tunneling electrons.
As such, the technique might be useful to address the role of different modes in cuprates. Motivated by the progress of elastic FT-STM technique, earlier few of us (JXZ, QS, AVB) have suggested an FT-IETS technique, Ref. 61, in a specific model that describes the Fourier transformed local density of states (LDOS) in a $d$-wave superconductor with the electronic coupling to the $(\pi, \pi)$ spin resonance mode. Here we go beyond the previous analysis and address the question of what consequences the electron self-energy renormalization will have on the tunneling characteristics for a number of collective modes broadly considered in the literature: the $B_{1 g}$ and breathing phonon modes, as well as the $(\pi, \pi)$ spin resonance mode. The central quantity we will focus on in our analysis is energy derivative of the FT LDOS, $\rho^{\prime}(\mathbf{q}, E)$. This quantity corresponds to $\frac{d^{2} I}{d V^{2}}(\mathbf{q}, e V)$ measured by the FTSTM, where $I$ is the local tunneling current and $V$ the voltage bias. Here we are not concerned with the mechanism of the superconductivity in the cuprates. Instead we assume from the outset a $d$-wave channel effective pairing interaction and study the additional effects due to the electronic coupling to various bosonic modes, including the $B_{1 g}$ and breathing phonon modes, and the $(\pi, \pi)$ spin resonance mode. A comparison of the calculated momentum transfer structure with the FT-STM measurement may shed new light on which type of bosonic mode the electron excitations are coupled to strongly.

The rest of the paper is outlined as follows: In Sec. I we develop a theoretical model in which the electrons are coupled to bosonic modes. We consider three types of modes: the $B_{1 g}$ and breathing phonon modes with possible coupling matrix elements, and the $(\pi, \pi)$ spin resonance mode. Additional weak disorder is used as a marker so that the momentum transfer can be investigated. In Sec. III the numerical results for the FT spectrum of the energy derivative LDOS are presented. Sec. IV] contains some concluding remarks.

\section{THEORETICAL MODEL}

We start with a BCS-type model to describe twodimensional electrons with a $d$-wave pairing symmetry, which is relevant to high-temperature cuprates:

$$
\mathcal{H}_{B C S}=\sum_{\mathbf{k}, \sigma} \xi_{\mathbf{k}} c_{\mathbf{k} \sigma}^{\dagger} c_{\mathbf{k} \sigma}+\sum_{\mathbf{k}}\left(\Delta_{\mathbf{k}} c_{\mathbf{k} \uparrow}^{\dagger} c_{-\mathbf{k} \downarrow}^{\dagger}+\Delta_{\mathbf{k}}^{*} c_{-\mathbf{k} \downarrow} c_{\mathbf{k} \uparrow}\right),
$$

where $c_{\mathbf{k} \sigma}^{\dagger}\left(c_{\mathbf{k} \sigma}\right)$ creates (annihilates) a conduction electron of spin $\sigma$ and wavevector $\mathbf{k}$. The quantity $\xi_{\mathbf{k}}$ is the normal state energy dispersion. We adopt a sixparameter fit to the band structure used previously for optimally doped Bi-2212 systems, $\stackrel{62}{,}$ having the form

$$
\begin{aligned}
\xi_{\mathbf{k}}= & -2 t_{1}\left(\cos k_{x}+\cos k_{y}\right)-4 t_{2} \cos k_{x} \cos k_{y} \\
& -2 t_{3}\left(\cos 2 k_{x}+\cos 2 k_{y}\right) \\
& -4 t_{4}\left(\cos 2 k_{x} \cos k_{y}+\cos k_{x} \cos 2 k_{y}\right) \\
& -4 t_{5} \cos 2 k_{x} \cos 2 k_{y}-\mu
\end{aligned}
$$


where $t_{1}=1, t_{2}=-0.2749, t_{3}=0.0872, t_{4}=0.0938$, $t_{5}=-0.0857$, and $\mu=-0.8772$. Unless specified explicitly, the energy is measured in units of $t_{1}$ hereafter.

As in previous works, 26.54.55.61 we assume that an effective $d$-wave pairing interaction has pre-existed from certain many-body effects. Therefore, even before the electronic coupling to the bosonic excitations, the $d$-wave superconducting order has already been established and the corresponding order parameter is given by:

$$
\Delta_{\mathrm{k}}=\frac{\Delta_{0}}{2}\left(\cos k_{x}-\cos k_{y}\right) \text {. }
$$

The additional renormalization comes from the additional electron-bosonic mode interaction.

By introducing a two-component spinor operator, one defines a matrix Green's function in the Nambu space. The bare Green's function in the assumption of a real $d$-wave pair potential reads

$$
\hat{\mathcal{G}}_{0}^{-1}\left(\mathbf{k} ; i \omega_{n}\right)=\left(\begin{array}{cc}
i \omega_{n}-\xi_{\mathbf{k}} & -\Delta_{\mathbf{k}} \\
-\Delta_{\mathbf{k}} & i \omega_{n}+\xi_{\mathbf{k}}
\end{array}\right) .
$$

Here $\omega_{n}=(2 n+1) \pi T$ is the fermionic Matsubra fre- quency.

\section{A. Coupling to the collective modes}

As mentioned before, there are many bosonic collective modes existing in cuprates. Here we focus on the out-ofplane out-of-phase buckling $\left(B_{1 g}\right)$ and the in-plane halfbreathing phonon modes associated with the motion of the $\mathrm{O}$ ions, and the $(\pi, \pi)$ spin resonance mode.

We model the electronic coupling to the phonon modes by

$$
\mathcal{H}_{e l-p h}=\frac{1}{\sqrt{N_{L}}} \sum_{\substack{\mathbf{k}, \mathbf{q} \\ \sigma}} g_{\nu}(\mathbf{k}, \mathbf{q}) c_{\mathbf{k}+\mathbf{q}, \sigma}^{\dagger} c_{\mathbf{k} \sigma} A_{\nu, \mathbf{q}}
$$

where $N_{L}$ is the number of lattice sites, $A_{\nu, \mathbf{q}}=b_{\nu \mathbf{q}}+b_{\nu,-\mathbf{q}}^{\dagger}$ with $b_{\nu \mathbf{q}}^{\dagger}\left(b_{\nu \mathbf{q}}\right)$ creating (annihilating) one phonon of type $\nu$ (representing $B_{1 g}$ or breathing mode) and wavevector q. We consider two types of the coupling matrix elements. The first type are both $\mathbf{q}$ and $\mathbf{k}$ dependent:

$$
\begin{aligned}
g_{B_{1 g}}(\mathbf{k}, \mathbf{q}) & =\frac{g_{0}}{\sqrt{M(\mathbf{q})}}\left\{\phi_{x}(\mathbf{k}) \phi_{x}(\mathbf{k}+\mathbf{q}) \cos \left(q_{y} / 2\right)-\phi_{y}(\mathbf{k}) \phi_{y}(\mathbf{k}+\mathbf{q}) \cos \left(q_{x} / 2\right)\right\} \\
g_{b r}(\mathbf{k}, \mathbf{q}) & =g_{0} \sum_{\alpha=x, y}\left\{\phi_{b}(\mathbf{k}+\mathbf{q}) \phi_{\alpha}(\mathbf{k}) \cos \left[\left(k_{\alpha}+q_{\alpha}\right) / 2\right]-\phi_{b}(\mathbf{k}) \phi_{\alpha}(\mathbf{k}+\mathbf{q}) \cos \left(k_{\alpha} / 2\right)\right\}
\end{aligned}
$$

where $M(\mathbf{q})=\left[\cos ^{2}\left(q_{x} / 2\right)+\cos ^{2}\left(q_{y} / 2\right)\right] / 2$, and

$$
\begin{aligned}
\phi_{x} & =\frac{i}{\mathcal{N}_{\mathbf{k}}}\left[\xi_{\mathbf{k}} t_{x, \mathbf{k}}-t_{x y, \mathbf{k}} t_{y, \mathbf{k}}\right], \\
\phi_{y} & =\frac{i}{\mathcal{N}_{\mathbf{k}}}\left[\xi_{\mathbf{k}} t_{y, \mathbf{k}}-t_{x y, \mathbf{k}} t_{x, \mathbf{k}}\right], \\
\phi_{b} & =\frac{1}{\mathcal{N}_{\mathbf{k}}}\left[\xi_{\mathbf{k}}^{2}-t_{x y, \mathbf{k}}^{2}\right],
\end{aligned}
$$

with $\mathcal{N}_{\mathbf{k}}=\left\{\left[\xi_{\mathbf{k}}^{2}-t_{x y, \mathbf{k}}^{2}\right]^{2}+\left[\xi_{\mathbf{k}} t_{x, \mathbf{k}}-t_{x y, \mathbf{k}} t_{y, \mathbf{k}}\right]^{2}+\right.$ $\left.\left[\xi_{\mathbf{k}} t_{y, \mathbf{k}}-t_{x y, \mathbf{k}} t_{x, \mathbf{k}}\right]^{2}\right\}^{1 / 2}, t_{\alpha, \mathbf{k}}=-2 t_{1} \sin \left(k_{\alpha} / 2\right)$ and $t_{x y, \mathbf{k}}=-4 t_{2} \sin \left(k_{x} / 2\right) \sin \left(k_{y} / 2\right)$. The $\mathbf{k}$ dependence has been argued to be crucial in the interpretation of ARPES data. ${ }^{12.55}$ The second type has only $\mathbf{q}$ dependence 21.54 .63 .64 .65 .66

$$
\begin{gathered}
\left|g_{B_{1 g}}(\mathbf{k}, \mathbf{q})\right|^{2}=\left|g_{0}\right|^{2}\left[\cos ^{2}\left(\frac{q_{x}}{2}\right)+\cos ^{2}\left(\frac{q_{y}}{2}\right)\right], \\
\left|g_{b r}(\mathbf{k}, \mathbf{q})\right|^{2}=\left|g_{0}\right|^{2}\left[\sin ^{2}\left(\frac{q_{x}}{2}\right)+\sin ^{2}\left(\frac{q_{y}}{2}\right)\right] .
\end{gathered}
$$

This type of $\mathbf{q}$ has been the focus before in the context

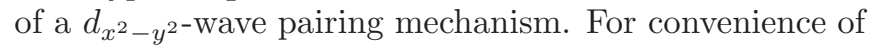

notation, we refer to the phonon modes with the first type of coupling as $B_{1 g}-\mathrm{I}$ and $b r$-I ones, while those with the second type of coupling as $B_{1 g}$-II and $b r$-II ones.

The electronic coupling to the $(\pi, \pi)$ spin resonance mode is modeled as:

$$
\mathcal{H}_{e l-s p}=\frac{g_{0}}{2 N_{L}} \sum_{\substack{\mathbf{k}, \mathbf{q} \\ \sigma, \sigma^{\prime}}} c_{\mathbf{k}+\mathbf{q}, \sigma}^{\dagger}\left(\mathbf{S}_{\mathbf{q}} \cdot \boldsymbol{\sigma}_{\sigma \sigma^{\prime}}\right) c_{\mathbf{k}, \sigma^{\prime}},
$$

where $\mathbf{S}$ is the spin operator for the $(\pi, \pi)$ mode, $\boldsymbol{\sigma}$ is the Pauli matrix vector.

We calculate the electronic self-energy due to the electron-bosonic excitation coupling up to the second order in the coupling matrix elements. For the electronphonon coupling, the self-energy is given as:

$$
\begin{aligned}
\hat{\Sigma}\left(\mathbf{k} ; i \omega_{n}\right)= & -\frac{T}{N_{L}} \sum_{\mathbf{q}, \nu} \sum_{\Omega_{m}} g_{\nu}(\mathbf{k}-\mathbf{q}, \mathbf{q}) g_{\nu}(\mathbf{k},-\mathbf{q}) \\
& \times \mathcal{D}_{\nu}\left(\mathbf{q} ; i \Omega_{m}\right) \hat{\tau}_{3} \hat{\mathcal{G}}_{0}\left(\mathbf{k}-\mathbf{q} ; i \omega_{n}-i \Omega_{m}\right) \hat{\tau}_{3}
\end{aligned}
$$

where $\Omega_{m}=2 m \pi T$ is the bosonic Matsubra frequency, $\hat{\tau}_{3}$ is the third component of the Pauli matrix in the 
Nambu space, the quantity $\mathcal{D}_{\nu}\left(\mathbf{q} ; i \Omega_{m}\right)$ is the Fourier transform of the phonon Green's function $\mathcal{D}_{\nu}(\mathbf{q} ; \tau)=$ $-\left\langle T_{\tau}\left[A_{\nu, \mathbf{q}}(\tau) A_{\nu,-\mathbf{q}}(0)\right]\right\rangle$ and is taken as

$$
\mathcal{D}_{\nu}\left(\mathbf{q} ; i \Omega_{m}\right)=\frac{1}{2}\left[\frac{1}{i \Omega_{m}-\Omega_{\nu}}-\frac{1}{i \Omega_{m}+\Omega_{\nu}}\right],
$$

with $\Omega_{\nu}$ being the resonance frequency of the phonon modes.

For the electron- $(\pi, \pi)$-resonance spin-fluctuation coupling, the self-energy is given as: ${ }^{61}$

$\hat{\Sigma}\left(\mathbf{k} ; i \omega_{n}\right)=-\frac{3 g_{0}^{2} T}{4 N_{L}} \sum_{\mathbf{q}} \sum_{\Omega_{m}} \chi\left(\mathbf{q} ; i \Omega_{m}\right) \hat{\mathcal{G}}_{0}\left(\mathbf{k}-\mathbf{q} ; i \omega_{n}-i \Omega_{m}\right)$,

where $\chi\left(\mathbf{q} ; i \Omega_{m}\right)$ is the Fourier transform of the spinspin correlation function, $\chi(\mathbf{q} ; \tau)=-\left\langle T_{\tau}\left[S_{\mathbf{q}}^{z}(\tau) S_{-\mathbf{q}}^{z}(0)\right]\right\rangle$ dynamical spin susceptibility. We treat the susceptibility in a phenomenological form: 26

$$
\chi\left(\mathbf{q} ; i \Omega_{m}\right)=-\frac{f(\mathbf{q})}{2}\left[\frac{1}{i \Omega_{m}-\Omega_{0}}-\frac{1}{i \Omega_{m}+\Omega_{0}}\right] .
$$

Here the spin resonance mode energy is also denoted by $\Omega_{0}$. The quantity $f(\mathbf{q})$ describes the momentum dependence of the mode and is assumed to be enhanced at the $\mathbf{Q}=(\pi, \pi)$ point. Using the correlation length $\xi_{s f}$ (chosen to be 2 here), it can be written as

$$
f(\mathbf{q})=\frac{1}{1+4 \xi_{s f}^{2}\left[\cos ^{2} \frac{q_{x}}{2}+\cos ^{2} \frac{q_{y}}{2}\right]} .
$$

The form of the dynamic susceptibility as given by Eq. (17) is especially suitable for the optimally doped $\mathrm{YBa}_{2} \mathrm{Cu}_{3} \mathrm{O}_{6+y}$ (YBCO) compounds in the superconducting phase, where the observed neutron resonance peak is almost resolution-limited in energy and fairly sharp in wavevector. The resonance peak in BSCCO is broadened in both energy and wavevector. In addition, given that the peak in BSCCO is still quite sharp in energy, we expect that the energy broadening of the resonance mode is not important for the present study. We have also neglected the incommensurate peaks seen in the inelastic neutron scattering experiments in YBCO (the part that disperses "downward" away from the resonance peak), 43,67,68,69,70 since their spectral weight is significantly smaller than that of the resonance mode.

The dressed electron Green's function $\mathcal{G}$, due to the renormalization effect of bosonic excitations, is given by:

$$
\hat{\mathcal{G}}^{-1}\left(\mathbf{k} ; i \omega_{n}\right)=\hat{\mathcal{G}}_{0}^{-1}\left(\mathbf{k} ; i \omega_{n}\right)-\hat{\Sigma}\left(\mathbf{k} ; i \omega_{n}\right) .
$$

To study the momentum transfer between the bosonic excitation renormalized electronic states, additional impurities or defects are required to scatter the electrons. The scattering from impurities is described by,

$$
H_{i m p}=\sum_{l \sigma} U_{l} c_{l \sigma}^{\dagger} c_{l \sigma}
$$

where $U_{l}$ is the strength of the zero-ranged impurity scattering potential at the $l$-th site. For simplicity, we consider only the case of nonmagnetic scattering and assume the scattering potential from all these impurities are identical, i.e., $U_{l}=U_{0}$. The full Green's function satisfies the following equation of motion:

$$
\hat{G}\left(i, j ; i \omega_{n}\right)=\hat{\mathcal{G}}\left(i, j ; i \omega_{n}\right)+\sum_{l} U_{l} \hat{\mathcal{G}}\left(i, l ; i \omega_{n}\right) \hat{\tau}_{3} \hat{G}\left(l, j ; i \omega_{n}\right) .
$$

Since we are most interested in effects of the electron collective mode coupling, it is desirable to keep as small as possible the disturbance (i.e., quantum interference, formation of virtual resonance etc.) arising from the quasiparticle scattering off the impurities themselves. This suggests to consider a dilute concentration of weak impurities. In this limit, the Born approximation is applicable, and one arrives at:

$$
\hat{G}\left(i, j ; i \omega_{n}\right)=\hat{\mathcal{G}}\left(i, j ; i \omega_{n}\right)+U_{0} \sum_{l} \hat{\mathcal{G}}\left(i, l ; i \omega_{n}\right) \hat{\tau}_{3} \hat{\mathcal{G}}\left(l, j ; i \omega_{n}\right)
$$

Due to the impurity scattering, the correction to the local density of states (LDOS) at the $i$-th site, summed over two spin components, is

$\delta \rho\left(\mathbf{r}_{i}, E\right)=-\frac{2 U_{0}}{\pi} \sum_{l} \operatorname{Im}\left[\hat{\mathcal{G}}(i, l ; E+i \gamma) \hat{\tau}_{3} \hat{\mathcal{G}}(l, i ; E+i \gamma)\right]_{11}$

\section{B. Fourier Transform}

The local density of states is proportional to the local differential tunneling conductance (i.e., $d I / d V$ ). To look into the renormalization effect of collective bosonic excitations in the STM, the energy derivative of the LDOS, corresponding to the derivative of the local differential tunneling conductance (i.e., $d^{2} I / d V^{2}$ ), is more favorable to enhance the signal. For a fixed value of energy, one first gets a set of $\delta \rho^{\prime}(i, E)$ (the prime / means the energy derivative) in real space, and then performs the Fourier transform:

$$
\delta \rho^{\prime}(\mathbf{q}, E)=\sum_{i} \delta \rho^{\prime}\left(\mathbf{r}_{i}, E\right) e^{-i \mathbf{q} \cdot \mathbf{r}_{i}}
$$

to obtain a map of the Fourier spectrum in $\mathbf{q}$ space,

$$
P(\mathbf{q}, E)=\left|\delta \rho^{\prime}(\mathbf{q}, E)\right| .
$$

One can also prove that the relation between $\delta \rho(\mathbf{q}, E)$ and that due to a single impurity $\delta \rho_{\text {single }}(\mathbf{q}, E)$ :

$$
\delta \rho^{\prime}(\mathbf{q}, E)=F(\mathbf{q}) \delta \rho_{\text {single }}^{\prime}(\mathbf{q}, E)
$$

where $F(\mathbf{q})$ is the form factor for the spatial distribution of weak impurities $[F(\mathbf{q})=1$ for the case of a single impurity]. 


\begin{tabular}{ccccc}
\hline \hline$B_{1 g}$-I mode & $b r$-I mode & $B_{1 g}$-II mode & $b r$-II mode & $(\pi, \pi)$ mode \\
\hline 1.05 & 1.48 & 0.75 & 0.75 & 2.30 \\
\hline \hline
\end{tabular}

TABLE I: The calibrated value of the coupling strength $g_{0}$ for different type of collective modes.

\section{RESULTS AND DISCUSSIONS}

For the numerical calculation, we take the superconducting energy gap $\Delta_{0}=0.1$, the frequency of all collective modes $\Omega_{0}=0.15$. A quasiparticle lifetime broadening of $\gamma=0.005$ is used. A weak impurity scattering strength $U_{0}=0.1$. We take a large system size $N_{L}=1024 \times 1024$ to achieve the high-momentum and energy resolution. The Fourier spectrum $P(\mathbf{q}, E)$ is then constructed from $\delta \rho^{\prime}\left(\mathbf{r}_{i} ; E\right)$ within a given window of size $61 \times 61$ centering the single impurity. We choose the coupling strength for all three types of collective modes in such a way that at the Fermi energy $E=0$, the frequency renormalization factor $Z$ appearing in the self energy

$\hat{\Sigma}\left(\mathbf{k} ; i \omega_{n}\right)=i \omega_{n}\left[1-Z\left(\mathbf{k} ; i \omega_{n}\right)\right] \hat{\tau}_{0}+\chi\left(\mathbf{k} ; i \omega_{n}\right) \hat{\tau}_{3}+\Phi\left(\mathbf{k} ; i \omega_{n}\right) \hat{\tau}_{1}$

has the same real-part value for the $B_{1 g}$-I, $B_{1 g}$-II, $b r$ II phonon modes, and $(\pi, \pi)$-resonance spin fluctuation modes at the $M$ point, while for the $b r$-I phonon mode at the wave-vector $(\pi / 2, \pi / 2)$. The calibrated value of the coupling strength $g_{0}$ for all these collective modes is summarized in Table
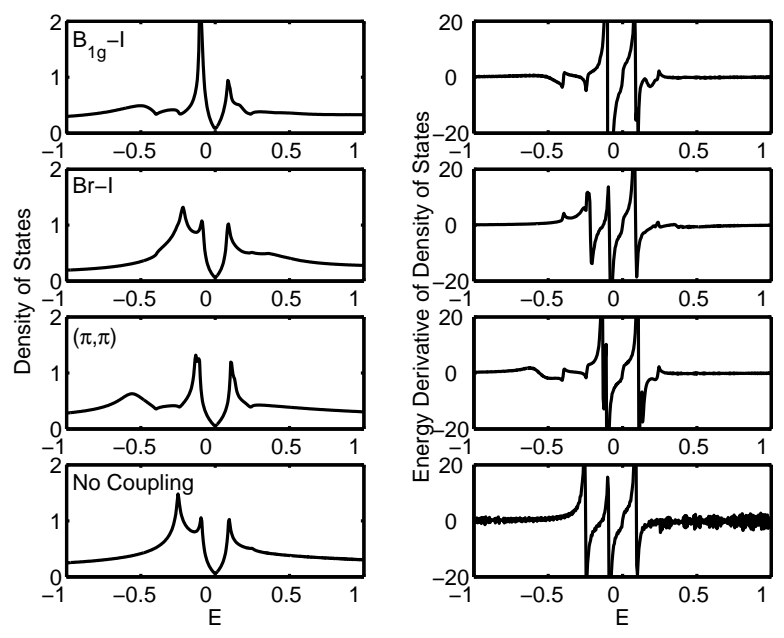

FIG. 1: Density of states (left column) and its energy derivative (right column) as a function of energy for a clean $d$-wave superconductor with the electronic coupling to $B_{1 g}$-I, br-I, and $(\pi, \pi)$ spin resonance modes. The case of no mode coupling is also shown for comparison.

We present in Fig. 11 the results of the DOS and its energy derivative as a function of energy for a clean (i.e., $\left.U_{0}=0\right) d$-wave superconductor with the electronic coupling to the $B_{1 g}$-I, br-I, $(\pi, \pi)$ spin resonance modes. For comparison, the DOS for the case of no mode coupling is also shown. When there is no electron-mode coupling, there is a van Hove singularity peak appearing outside the superconducting gap edge. When the electrons are coupled to the $B_{1 g}$ and $(\pi, \pi)$-spin resonance modes, the van Hove singularity peak is strongly suppressed. Instead, one sees a dip structure following the coherent peak at the gap edge. The distance between this dip and the coherent peak defines the resonance energy $\Omega_{0}$. However, there is very little suppression when the electrons are coupled to the $b r$-I mode. The planar tunneling experiments indeed observed the peak-dip structure rather than the peak-peak (van Hove singularity) structure. For the band-structure parametrization we have adopted, this implies that the electronic coupling to the collective modes must exist, and the $B_{1 g}$ and $(\pi, \pi)$ spin resonance mode are the most promising candidates. Unfortunately, as shown in Fig. 11 the dip structure due to the coupling to the $B_{1 g}$ and $(\pi, \pi)$ spin resonance mode is almost identical. It would be very challenging to distinguish between these two modes in the planar tunneling experiments, which is measuring the momentum averaged DOS. Therefore, we propose to look at the momentum transfer structure between the band renormalized states, which can be measured by the FT-STM. To achieve this goal, we need to have a signal strong enough to be detectable in STM experiments. The derivative of the DOS, $\rho^{\prime}(E)$, would serve the purpose. As shown in the right column of Fig. 1] when the electrons are coupled to the $B_{1 g}$ and spin resonance modes, there is a strong peak structure at $E=-\left(\Delta_{0}+\Omega_{0}\right)$ exhibiting in the $\rho^{\prime}(E)$ spectrum, which has a one-to-one correspondence to the dip structure in $\rho(E)$ itself.
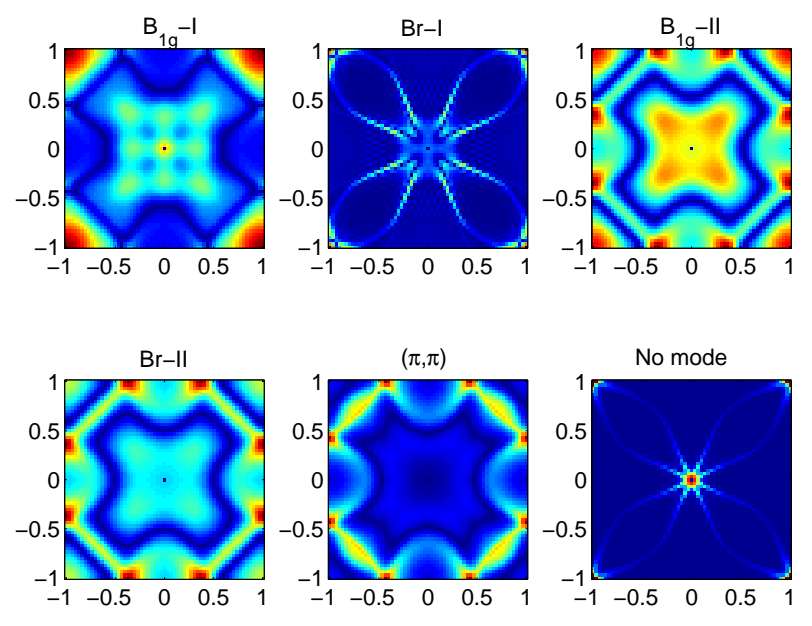

FIG. 2: The Fourier spectral weight of the energy derivative of the LDOS at $E=-\left(\Delta_{0}+\Omega_{0}\right)$ for a $d$-wave superconductor with the electronic coupling to the $B_{1 g}$, br-I, $B_{1 g}$-II, br-II, spin resonace modes. For comparison, the quantity is also shown for the case of no mode coupling.

In Fig. 2 we present the results of the Fourier spectrum of the derivative of the LDOS, $\rho^{\prime}(\mathbf{q}, E)$, at the energy $E=-\left(\Delta_{0}+\Omega_{0}\right)$ for a $d$-wave superconductor with 
the electronic coupling to the collective modes. For comparison, the same quantity is also shown (last panel) for the case of no mode coupling. Note that the case without the mode coupling, the energy $\Omega_{0}$ has no special meaning in the context of the electronic properties, and the energy $E=-\left(\Delta_{0}+\Omega_{0}\right)$ is chosen merely for comparison to the case of mode coupling. The main results are: For all cases, there are strong intensity at the large momentum transfer near $(\pi, \pi)$. For the cases of the $b r$-I coupling

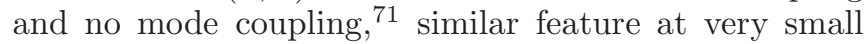
momentum transfer is obtained, relating to the fact that the DOS spectrum in two cases (see Fig. 11) is similar to each other. For the cases of the coupling to the $B_{1 g}$-II and $b r$-II modes, there are intensity peaks at a finite momentum transfer along the diagonals. For the case of the coupling to the spin resonance mode, no strong feature is obtained at the intermediate value of momentum transfer, which is consistent with our previous calculation for this specific case $\frac{61}{6}$ For the case of the coupling to the $B_{1 g}$-I phonon mode, there exists not only the intensity peaks with a momentum transfer along the diagonal but also the ones with a momentum transfer along the bond directions of a square lattice.
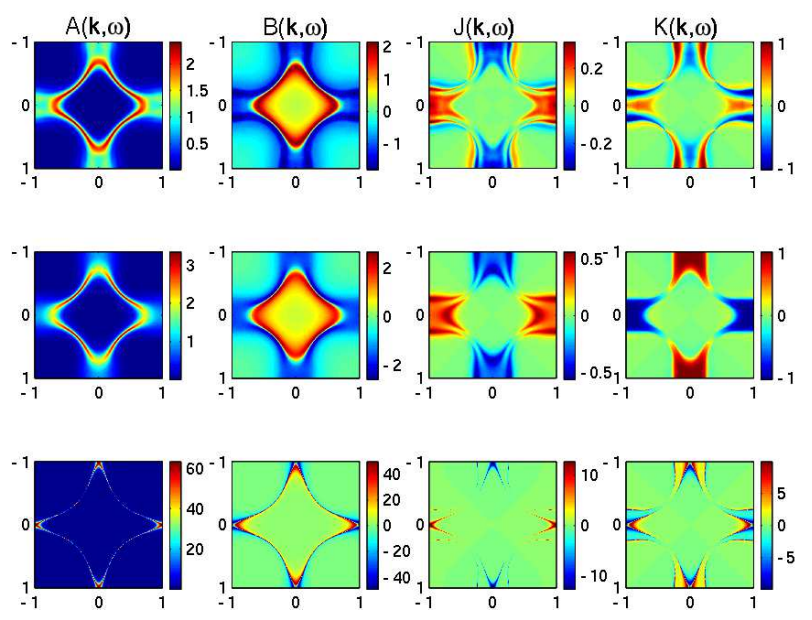

FIG. 3: The imaginary and real parts of the single particle and anomalous Green's function, $A(\mathbf{k} ; E), B(\mathbf{k} ; E), J(\mathbf{k} ; E)$, and $K(\mathbf{k} ; E)$ [as defined by Eqs. 29) - 32] , at the energy $E=$ $-\left(\Delta_{0}+\Omega_{0}\right)$ for the electronic coupling to the $B_{1 g}$ phonon (first row), spin resonance mode (second row), and the case of no mode coupling.

The different Fourier spectrum patterns come from the detailed renormalization of electronic structure by the coupling to these modes. To better understand these patterns, we turn to a detailed analytical form of the Fourier transform. By putting aside the external form factor associated with a specific configuration of weak disorder, the Fourier spectrum is determined uniquely by the electronic single particle Green's function and is found to be:

$$
\begin{gathered}
\delta \rho(\mathbf{q} ; E)=\frac{u_{0}}{N_{L}} \sum_{\mathbf{k}} \\
\{[A(\mathbf{k} ; E) B(\mathbf{k}+\mathbf{q} ; E)+B(\mathbf{k} ; E) A(\mathbf{k}+\mathbf{q} ; E)] \\
-[J(\mathbf{k} ; E) K(\mathbf{k}+\mathbf{q} ; E)+K(\mathbf{k} ; E) J(\mathbf{k}+\mathbf{q} ; E)]\}
\end{gathered}
$$

where

$$
\begin{aligned}
A(\mathbf{k} ; E) & =-\frac{2}{\pi} \operatorname{Im}\left[\mathcal{G}_{11}(\mathbf{k} ; E+i \gamma)\right], \\
B(\mathbf{k} ; E) & =\operatorname{Re}\left[\mathcal{G}_{11}(\mathbf{k} ; E+i \gamma)\right], \\
J(\mathbf{k} ; E) & =-\frac{2}{\pi} \operatorname{Im}\left[\mathcal{G}_{12}(\mathbf{k} ; E+i \gamma)\right], \\
K(\mathbf{k} ; E) & =\operatorname{Re}\left[\mathcal{G}_{12}(\mathbf{k} ; E+i \gamma)\right] .
\end{aligned}
$$

Here as shown in Eqs. (28) through (32), the Fourier spectrum is determined by the convolution of the imaginary ${ }^{72}$ and the real parts of the single-particle $\left(\mathcal{G}_{11}\right)$ and anomalous $\left(\mathcal{G}_{12}\right)$ Green's function in the superconducting state. The stronger intensity in $\delta \rho(\mathbf{q} ; E)$ (also $\left.\delta \rho^{\prime}(\mathbf{q} ; E)\right)$ will be contributed from the wave vector $\mathbf{q}$, which connects the simultaneously largest intensity in $A(\mathbf{k} ; E)$ and $B(\mathbf{k} ; E)$ maps, and in $J(\mathbf{k} ; E)$ and $K(\mathbf{k} ; E)$ maps. To be illustrative, we present in Fig. [3 those maps for the electronic coupling to the $B_{1 g^{-}} \mathrm{I}$ and spin resonance modes, and also for the case without the mode coupling at $E=-\left(\Delta_{0}+\Omega_{0}\right)$. Notice that the joint intensity of $J(\mathbf{k} ; E) K\left(\mathbf{k}^{\prime} ; E\right)$ are smaller by an overall factor of 10 than that of $A(\mathbf{k} ; E) B\left(\mathbf{k}^{\prime} ; E\right)$ and the Fourier spectrum is mostly determined by the latter product. For the case of no mode coupling, the strongest weight in these quantities are located at the $M$ points of the first Brillouin zone, which leads to the strongest intensity of the Fourier spectrum with the momentum transfer $\mathbf{q}=(\pi, \pi)$ and the zero momentum transfer $\mathbf{q}=0$. If the electrons are coupled to the $B_{1 g}$-I phonon mode, besides the strongest intensity on the closed ridges in $A(\mathbf{k} ; E)$ and $B(\mathbf{k} ; E)$ [red area], there are also moderately strong intensity on the two split beams around the $M$ points in these two maps [bright green in the former and dark blue in the latter]. The intensity $A(\mathbf{k} ; E) B\left(\mathbf{k}^{\prime} ; E\right)$ connected by $\mathbf{q}=\mathbf{k}^{\prime}-\mathbf{k}$ with $\mathbf{k}$ and $\mathbf{k}^{\prime}$ located at the ends of these beams becomes stronger. These wave vectors are just those in the $B_{1 g^{-}}$ I panel of Fig. 2 at which the Fourier spectrum exhibits peaks [green spots]. However, if the electrons are coupled to the spin resonance mode, no such beams exist, which explains the lack of peaks in Fourier spectrum [see the $(\pi, \pi)$ panel in Fig. 2].

Experimentally, only the peaks in the Fourier spectrum with the momentum transfer along the bond direction has been observed so far ${ }^{73}$ The scenario that the electrons are coupled to the $B_{1 g}$ phonon mode and the coupling matrix is highly anisotropic (i.e., $B_{1 g}$-I mode) bears the closest resemblance to the experimental observation, though not in a full agreement. In Fig. 团 we present the energy evolution of the spectrum pattern for the electronic coupling to the $B_{1 g^{-}}$I mode. It shows that the characteristic momentum transfer wave vector decreases 

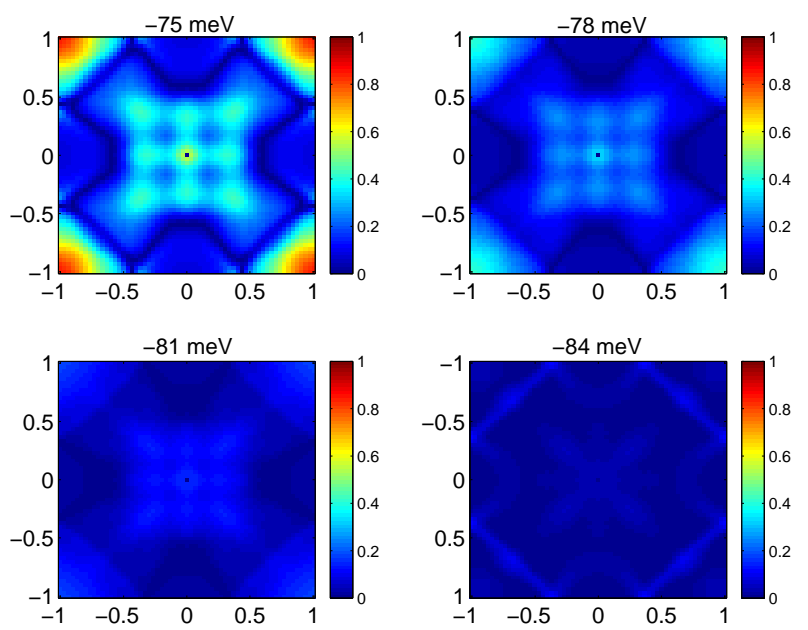

FIG. 4: The Fourier spectrum of the derivative of the LDOS is shown at the various values of the energy for the case of the electronic coupling to the $B_{1 g}$ phonon mode. Here the energy has been measured by scaling $\Delta_{0}=30 \mathrm{meV}$.

slightly with the increased energy. However, the intensity at these wave vectors decreases rapidly when the energy moves away from the action point $-\left(\Delta_{0}+\Omega_{0}\right)$. This is also not inconsistent with the experiment.

\section{CONCLUDING REMARKS}

There is considerable evidence that numerous ARPES and tunneling data can be interpreted in terms of the electronic coupling to bosonic modes with energy about $40 \mathrm{meV}$. Possible candidates for this mode are the $(\pi, \pi)$ spin resonance mode and various phonon modes. The planar junction tunneling has provided an accurate measurement of the energy scale of this mode. However, since the structure in the tunneling spectra due to the electronic coupling to $B_{1 g}$ phonon mode or to the $(\pi, \pi)$ spin resonance mode does not have much difference, it is very difficult to determine the nature of the mode.

In this paper, we have analyzed the Fourier spectra of the energy derivative LDOS to investigate the momentum transfer structure arising from the electronic coupling to these different modes. This quantity nicely complements ARPES for the understanding of the electronic responses to these modes. It can now be obtained from the FT-STM experiments with elevated spatial resolution. In general, we found that the detailed momentum dependence of the coupling matrix element strongly influences the electronic properties. In particular, we have shown that if the $B_{1 g}$ or breathing modes are coupled to the electrons with only a $\mathbf{q}$ dependence, the spectrum displays the peak structure along the diagonals with a small momentum transfer while the $(\pi, \pi)$ mode coupling does not produce much weight at the small momentum transfer region. On the other hand, if the electrons are coupled to the $B_{1 g}$ phonon mode with a matrix element that depends not only on $\mathbf{q}$ but also on $\mathbf{k}$, the peak structure with a small momentum transfer can also appear along the $\mathrm{Cu}-\mathrm{O}$ bond directions of the $\mathrm{CuO}_{2}$ plane. Recent FT-STM experiments have indeed found a peak at a bond-directed momentum transfer.

Our calculations also show the structures at large momentum transfer for all cases of the electron-collective mode coupling. In other words, both types (electronic and phononic) of mode couplings produce structures near $(\pi, \pi)$, but only a coupling to the phonon modes yields additional structures at the small momentum transfers. No peak structures near $(\pi, \pi)$ have been observed in the experiments. The situation is somewhat similar to the elastic scattering case (i.e., in the absence of the collective mode coupling), where similar structures near $(\pi, \pi)$ also appear in the theoretical spectra ${ }^{74.75}$ but are not observed experimentally. It is likely that the lack of structures at large momentum-transfer in the elastic and inelastic experiments has a common origin. One possibility has to do with strong inhomogeneities, which may give rise to a dominant forward scattering and make only the structures at small momentum transfers observable. This amounts to the following form factor for the weak disorder configuration:

$$
F(\mathbf{q})=\frac{1}{1+r_{c}\left[\sin ^{2}\left(q_{x} / 2\right)+\sin ^{2}\left(q_{y} / 2\right)\right]},
$$

where the parameter $r_{c}$ controls the range of the forward scattering in the $\mathbf{q}$ space. The overall modulation in the Fourier spectrum of the derivative LDOS $\delta \rho^{\prime}(\mathbf{q}, \omega) \propto F(\mathbf{q})$ will be confined to small momenta if $F(\mathbf{q})$ is.

Finally, several remarks are in order: (i) The electroncollective mode coupling we have considered preserves the translational symmetry. Fourier spectra were studied after introducing elastic impurity scattering with weak scattering potential. Alternatively, local vibrational mode scattering will not only provide an inelastic scattering channel but also will break the translational symmetry at the beginning 76 To fully understand the possible FT-STM experiments, it would be instructive to consider the electronic coupling to the distributed local vibrational modes. We leave this problem for separate analysis. (ii) There has also be increased interest in the quasiparticle scattering from the $\tau_{1}$ impurities 77 It would be very helpful to study the the FT spectrum through the $\tau_{1}$ scatterers. We delay this investigation to a future publication.

\section{Acknowledgments}

We thank D.-H. Lee, N. Nagaosa, M. R. Norman, D. J. Scalapino, and Z. X. Shen for very useful discussions. This work was supported by the US DOE (J.X.Z. and A.V.B.), the NSERC, the Office of Naval Research under Grant No. N00014-05-1-0127, and the A. von Humboldt 
Foundation (T.P.D.), the NSF under Grant No. DMR0424125 and the Robert A. Welch Foundation (Q.S.), the Office of Naval Research under grant N00014-03-1-
0674, the NSF under Grant No. DMR-9971502, the NSFITR FDP-0205641, and the Army Research Office under Grant No. DAAD19-02-1-0043 (K.M., J.L., and J.C.D).
1 D. S. Dessau, B. O. Wells, Z.X. Shen, W. E. Spicer, A. J. Arko, R. S. List, D. B. Mitzi, A. Kapitulnik, Phys. Rev. Lett. 66, 2160 (1991).

2 H. Ding, A. F. Bellman, J. C. Campuzano, M. Randeria, M. R. Norman, T. Yokoya, T. Takahashi, H. KatayamaYoshida, T. Mochiku, K. Kadowaki, G. Jennings, and G. P. Brivio, Phys. Rev. Lett. 76, 1533 (1996).

3 J. C. Campuzano, H. Ding, M. R. Norman, H. M. Fretwell, M. Randeria, A. Kaminski, J. Mesot, T. Takeuchi, T. Sato, T. Yokoya, T. Takahashi, T. Mochiku, K. Kadowaki, P. Guptasarma, D. G. Hinks, Z. Konstantinovic, Z. Z. Li, and H. Raffy Phys. Rev. Lett. 83, 3709 (1999).

${ }^{4}$ P. V. Bogdanov, A. Lanzara, S. A. Kellar, X. J. Zhou, E. D. Lu, W. J. Zheng, G. Gu, J.-I. Shimoyama, K. Kishio, H. Ikeda, R. Yoshizaki, Z. Hussain, and Z. X. Shen, Phys. Rev. Lett. 85, 2581 (2000).

5 A. Kaminski, M. Randeria, J. C. Campuzano, M. R. Norman, H. Fretwell, J. Mesot, T. Sato, T. Takahashi, and K. Kadowaki, Phys. Rev. Lett. 86, 1070 (2001).

6 A. Lanzara, P. V. Bogdanov, X. J. Zhou, S. A. Kellar, D. L. Feng, E. D. Lu, T. Yoshida, H. Eisaki, A. Fujimori, K. Kishio, J.-I. Shimoyama, T. Noda, S. Uchida, Z. Hussain, Z.-X. Shen, Nature 412, 510 (2001).

7 P. D. Johnson, T. Valla, A. V. Fedorov, Z. Yusof, B. O. Wells, Q. Li, A. R. Moodenbaugh, G. D. Gu, N. Koshizuka, C. Kendziora, Sha Jian, and D. G. Hinks, Phys. Rev. Lett. 87, 177007 (2001).

8 X. J. Zhou, T. Yoshida, A. Lanzara, P. V. Bogdanov, S. A. Kellar, K. M. Shen, W. L. Yang, F. Ronning, T. Sasagawa, T. Kakeshita, T. Noda, H. Eisaki, S. Uchida, C. T. Lin, F. Zhou, J. W. Xiong, W. X. Ti, Z. X. Zhao, A. Fujimori, Z. Hussain, Z.-X. Shen, Nature 423, 398 (2003).

9 T. K. Kim, A. A. Kordyuk, S. V. Borisenko, A. Koitzsch, M. Knupfer, H. Berger, and J. Fink, Phys. Rev. Lett. 91, 167002 (2003).

10 A. D. Gromko, A. V. Fedorov, Y.-D. Chuang, J. D. Koralek, Y. Aiura, Y. Yamaguchi, K. Oka, Yoichi Ando, and D. S. Dessau, Phys. Rev. B 68, 174520 (2003).

11 T. Sato, H. Matsui, T. Takahashi, H. Ding, H.-B. Yang, S.-C. Wang, T. Fujii, T. Watanabe, A. Matsuda, T. Terashima, and K. Kadowaki, Phys. Rev. Lett. 91, 157003 (2003).

12 T. Cuk, F. Baumberger, D. H. Lu, N. Ingle, X. J. Zhou, H. Eisaki, N. Kaneko, Z. Hussain, T. P. Devereaux, N. Nagaosa, and Z.-X. Shen, Phys. Rev. Lett. 93, 117003 (2004).

13 Q. Huang, J. E. Zasadzinski, K. E. Gray, J. Z. Liu, and H. Claus, Phys. Rev. B 40, 9366 (1989).

14 Ch. Renner and Ø. Fischer, Phys. Rev. B 51, 9208 (1995).

15 Ch. Renner, B. Revaz, J.-Y. Genoud, and Ø. Fischer, J. Low Temp. Phys. 105, 1083 (1996).

16 Y. DeWilde, N. Miyakawa, P. Guptasarma, M. Iavarone, L. Ozyuzer, J. F. Zasadzinski, P. Romano, D. G. Hinks, C. Kendziora, G. W. Crabtree, and K. E. Gray, Phys. Rev. Lett. 80, 153 (1998).

17 D. Mandrus, L. Forro, D. Koller, and L. Mihaly, Nature 351, 460 (1991).
18 A. Yurgens, D. Winkler, T. Claeson, S.-J. Hwang, and J.H. Choy, Int. J. Mod. Phys. B 29-31, 3758 (1999).

19 J.F. Zasadzinski, L. Ozyuzer, N. Miyakawa, D. G. Hinks, K. E. Gray, Physica C 341-348, 867 (2000).

20 J. F. Zasadzinski, L. Ozyuzer, N. Miyakawa, K. E. Gray, D. G. Hinks, and C. Kendziora, Phys. Rev. Lett. 87, 067005 (2001).

21 T. Dahm, D. Manske, D. Fay, and L. Tewordt, Phys. Rev. B 54, 12006 (1996); T. Dahm, D. Manske, and L. Tewordt, Phys. Rev. B 58, 12454 (1998).

22 Z. X. Shen and J. R. Schrieffer, Phys. Rev. Lett. 78, 1771 (1997).

23 M. R. Norman, H. Ding, J. C. Campuzano, T. Takeuchi, M. Randeria, T. Yokoya, T. Takahashi, T. Mochiku, and K. Kadowaki, Phys. Rev. Lett. 79, 3506 (1997).

24 M. R. Norman and H. Ding, Phys. Rev. B 57, R11089 (1998).

25 Ar. Abanov and A. V. Chubukov, Phys. Rev. Lett. 83, 1652 (1999); Phys. Rev. B 61, R9241 (2000).

26 M. Eschrig and M. R. Norman, Phys. Rev. Lett. 85, 3261 (2000); Phys. Rev. B 67, 144503 (2003).

27 M. R. Norman, M. Eschrig, A. Kaminski, and J. C. Campuzano, Phys. Rev. B 64, 184508 (2001).

28 D. Manske, I. Eremin, and K. H. Bennemann, Phys. Rev. lett. 87, 177005 (2001).

29 Ar. Abanov, A. V. Chubukov, and J. Schmalian, Adv. Phys. 52, 119 (2003).

30 J. Rossat-Mignod, L. P. Regnault, C. Vettier, P. Bourges, P. Burlet, J. Bossy, J. Y. Henry, and G. Lapertot, Physica C 185-189, 86 (1991).

31 H. A. Mook, M. Yethiraj, G. Aeppli, T. E. Mason, and T. Armstrong, Phys. Rev. Lett. 70, 3490 (1993).

32 H. F. Fong, B. Keimer, P. W. Anderson, D. Reznik, F. Dogan, and I. A. Aksay, Phys. Rev. Lett. 75, 316 (1995); H. F. Fong, B. Keimer, D. Reznik, D. L. Milius, and I. A. Aksay, Phys. Rev. B 54, 6708 (1996).

33 P. Bourges, L. P. Regnault, J. Y. Henry, C. Vettier, Y. Sidis, and P. Burlet, Physica B 215, 30 (1995).

34 P. Bourges, L. P. Regnault, Y. Sidis, and C. Vettier, Phys. Rev. B 53, 876 (1996).

35 H. F. Fong, P. Bourges, Y. Sidis, L. P. Regnault, A. Ivanov, G. D. Gu, N. Koshizuka, and B. Keimer, Nature 398, 588 (1999);

36 P. Dai, M. Yethiraj, H. A. Mook, T. B. Linemer, and F. Dogan, Phys. Rev. Lett. 77, 5425 (1996).

37 H. F. Fong, B. Keimer, D. L. Milius, and I. A. Aksay, Phys. Rev. Lett. 78, 713 (1997).

38 P. Bourges, H. F. Fong, L. P. Regnault, J. Bossy, C. Vettier, D. L. Milius, I. A. Aksay, and B. Keimer, Phys. Rev. Lett. 78, 713 (1997).

39 P. Dai, H. A. Mook, S. M. Hayden, G. Aeppli, T. G. Perring, R. D. Hunt, and F. Dogan, Science 284, 1344 (1999).

40 H. F. He, Y. Sidis, P. Bourges, G. D. Gu, A. Ivanov, N. Koshizuka, B. Liang, C. T. Lin, L. P. Regnault, E. Schoenherr, and B. Keimer, Phys. Rev. Lett. 86, 1610 (2001).

${ }^{41}$ H. F. He, P. Bourges, Y. Sidis, C. Ulrich, L. P. Regnault, 
S. Pailhes, N. S. Berzigiarova, N. N. Kolesnikov, and B. Keimer, Science 295, 1045 (2002).

42 P. Bourges, in The Gap Symmetry and Fluctuations in High Temperature Superconductors, edited by J. Bok, G. Deutscher, D. Pavuna, and S. A. Wolf (Plenum, New York, 1998), p.349.

43 H. F. Fong, P. Bourges, Y. Sidis, L. P. Regnault, J. Bossy, A. Ivanov, D. L. Milius, I. A. Aksay, and B. Keimer, Phys. Rev. B 61, 14773 (2000).

44 P. Bourges, B. Keimer, L. P. Regnault, and Y. Sidis, J. Supercond. 13, 735 (2000).

45 P. Dai, H. A. Mook, R. D. Hunt, and F. Dogan, Phys. Rev. B 63, 054525 (2001).

46 B. Friedl, C. Thomsen, and M. Cardona, Phys. Rev. Lett. 65, 915 (1990).

47 N. Pyka, W. Reichardt, L. Pintschovius, G. Engel, J. RossatMignod, and J. Y. Henry, Phys. Rev. Lett. 70, 1457 (1993).

48 D. Reznik, B. Keimer, F. Dogan, and I. A. Aksay, Phys. Rev. Lett. 75, 2396 (1995).

49 S. L. Chaplot, W. Reichardt, L. Pintschovius, and N. Pyka, Phys. Rev. B 52, 7230 (1995).

50 R. J. McQueeney, Y. Petrov, T. Egami, M. Yethiraj, G. Shirane, and Y. Endoh, Phys. Rev. Lett. 82, 628 (1999); R. J. McQueeney, J. L. Sarrao, P. G. Pagliuso, P. W. Stephens, and R. Osborn, Phys. Rev. Lett. 87, 077001 (2001).

51 J.-H. Chung, T. Egami, R. J. McQueeney, M. Yethiraj, M. Arai, T. Yokoo, Y. Petrov, H. A. Mook, Y. Endoh, S. Tajima, C. Frost, and F. Dogan, Phys. Rev. B 67, 014517 (2003).

52 S. Sugai, H. Suzuki, Y. Takayanagi, T. Hosokawa, and N. Hayamizu, Phys. Rev. B 68, 184504 (2003).

${ }^{53}$ M. Opel, R. Hackl, T. P. Devereaux, A. Virosztek, A. Zawadowski, A. Erb, E. Walker, H. Berger, and L. Forró, Phys. Rev. B 60, 9836 (1999).

54 A. W. Sandvik, D. J. Scalapino, and N. E. Bickers, Phys. Rev. B 69, 094523 (2004).

55 T. P. Devereaux, T. Cuk, Z.-X. Shen, and N. Nagaosa, Phys. Rev. Lett. 93, 117004 (2004).

56 R. C. Jaklevic and J. Lambe, Phys. Rev. Lett. 17, 1139 (1966); D. J. Scalapino and S. M. Markus, Phys. Rev. Lett. 18, 459 (1967).

57 P. Hansma, Ed., Tunneling Spectroscopy: Capabilities, Application and New Techniques (Plenum Press, New York, 1982).

58 D. J. Scalapino, in "The Electron Phonon Interaction and Strong-Coupling Superconductors", in Superconductivity, Vol. 1, Chapter 10, p. 449, ed. R.D. Parks (Marcel Dekker, New York, 1969). See also W. L. McMillan and J. M. Rowell, "Tunneling and Strong Coupling Superconductivity", the same volume, Chapter 10, p. 561 and references therein.

59 J. E. Hoffman, K. McElroy, D.-H. Lee, K. M. Lang, H. Eisaki, S. Uchida, and J. C. Davis, Science 297, 1148
(2002).

60 K. McElroy, R. W. Simmonds, J. E. Hoffman, D. H. Lee, J. Orenstein, H. Eisaki, S. Uchida, J. C. Davis, Nature 422, 592 (2003).

61 J.-X. Zhu, J. Sun, Q. Si, and A. V. Balatsky, Phys. Rev. Lett. 92, 017002 (2004).

62 M. R. Norman, M. Randeria, H. Ding, and J. C. Campuzano, Phys. Rev. B 52, 615 (1995).

63 J. Song and J. F. Annett, Phys. Rev. B 51, 3840 (1995); 52, 6930(E) (1995).

64 D. J. Scalapino, J. Phys. Chem. Solids 56, 1669 (1995).

65 A. Nazarenko and E. Dagotto, Phys. Rev. B 53, R2987 (1996).

66 N. Bulut and D. J. Scalapino, Phys. Rev. B 54, 14971 (1996).

67 P. Dai, H. A. Mook, and F. Dogan, Phys. Rev. Lett. 80, 1738 (1998); H. A. Mook, P. Dai, R. D. Hunt, and F. Dogan, J. Phys. Chem. Solids 59, 2140 (1998); H. A. Mook, P. Dai, S. M. Hayden, G. Aeppli, T. G. Perring, and F. Dogan, Nature 395, 580 (1998).

68 M. Arai, T. Nishijima, Y. Endoh, T. Egami, S. Tajima, K. Tomimoto, Y. Shiohara, M. Takahashi, A. Garrett, and S. M. Bennington, Phys. Rev. Lett. 83, 608 (1999).

69 J. Brinckmann and P. A. Lee, Phys. Rev. Lett. 82, 2915 (1999);

70 Y.-J. Kao, Q. Si, and K. Levin, Phys. Rev. B 61, R11898 (2000); Y. Zha, K. Levin, and Q. Si, Phys. Rev. B 47, 9124 (1993).

71 Here, with the experiment of Ref. 73 in mind, we have done a Fourier transform of the LDOS in the entire field of view. If impurities are explicitly identified, as in the case of Zn-doped cuprates, a filtered Fourier transform can also be adopted. As discussed in Ref. 61, this procedure would consider the LDOS at the sites other than those of two diagonal strips; in other words, the Fourier transform would only incorporate sites from four disconnected trangles. Within this filtered Fourier transform procedure, the features near $(\pi, \pi)$ disappear in the absence of any mode coupling, but persist for all the cases involving a mode coupling.

72 Notice that the spectral weight $A(\mathbf{k}, \omega)$ as given by Eq. (29) is the quantity that the ARPES experiments can measure. The role of the $(\pi, \pi)$-resonance spin fluctuation and the phonon modes on the superconducting state spectral function has been studied previously. This function gives the information about the single particle energy dispersion in the first Brillouin zone in a homogeneous system.

73 J. Lee et al. (unpublished).

74 Q.-H. Wang and D.-H. Lee, Phys. Rev. B 67, 020511(R) (2003).

75 D. Zhang and C. S. Ting, Phys. Rev. B 67, 100506 (2003).

76 A. V. Balatsky, Ar. Abanov, and J.-X. Zhu, Phys. Rev. B 68, 214506 (2003), and references therein.

77 T. S. Nunner, B. M. Anderson, A. Melikyan, and P. J. Hirschfeld, cond-mat/0504693 (unpublished). 